\title{
Crystal Structure of Hybrid Dipeptide, Cytosinyl-L-tyrosine
}

\author{
Mitsunobu DoI, Hajime MiYako, Akiko Asano and Toshimasa IshIDA \\ Osaka University of Pharmaceutical Sciences, 4-20-1, Nasahara, Takatsuki, Osaka 569-1094, Japan
}

A series of hybrid dipeptides, the structure properties of which are similar to that of peptide nucleic acid (PNA), has been designed to investigate the cooperative interactions between the nucleic acid and polypeptide. ${ }^{1}$ The structure of dimeric cytosinyl-L-tryptophan (C-Trp) hexahydrate and the interaction mode between the cytosine base and the indol ring were previously reported $^{2}$, and it was suggested that the dipeptide containing cytosine and tryptophan selectively interacted with the 7-methylated guanine base. ${ }^{3}$ We have been focusing on the functional group of the amino acid incorporated in the nucleic acid base, and have now analyzed the structure of cytosinyl-L-tyrosine (C-Tyr) by the X-ray diffraction method to estimate the function of the phenol ring in the pyrimidine base.

The title compound (Fig.1) was synthesized by the coupling 1-ethylcarboxy-cytosine and tyrosine methyl ester, and crystals were obtained from an aqueous methanol solution. The intensity data were collected in the $\omega-2 \theta$ scan mode using graphite-monochromated $\mathrm{Cu}$ $\mathrm{K}_{\alpha}$ radiation $\lambda=1.5418 \AA$ ). Although the data were corrected for Lorentz and polarization factors, no absorption correction was applied. Table 1 summarizes the crystal and experimental data. Atoms were blocked into two parts in refinement cycles, and the positions and thermal factors of each block were refined in a single cycle. Non-hydrogen atoms were refined anisotropically and hydrogen atoms calculated at idealized positions were included in the calculation of the structure factors. The atomic parameters are given in Table 2 . The bond distances and selected torsion angles are given in Table 3.

There are two crystallographically independent molecules (Molecules $\mathbf{1}$ and $\mathbf{2}$ ) in the asymmetric unit (Fig. 2). The independent molecules are discriminated by the differences in the conformation of the tyrosine moiety: the torsion angles of Molecule 1/2 around C8-C9, N10-C10A and C12-C13 are 148.2(3)/125.7(3), $-98.4(3) /-73.7(3)$ and $-37.9(4) / 112.5(4)^{\circ}$, respectively

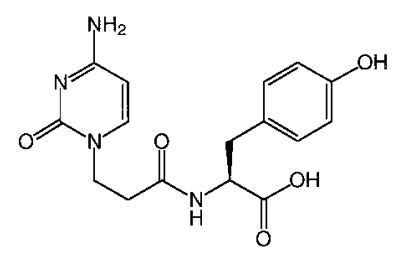

Fig. 1 Chemical structure of C-Tyr.
(Table 3). Both molecules are folded in a similar manner. Although the folded conformation results in access between the cytosine base and the phenol ring, no notable interactions are observed between the aromatic rings.

The C-terminal groups of tyrosine seem to be an unionized form $(-\mathrm{COOH})$ in both molecules. The cytosine bases interact with these carboxyl groups between the independent molecules: N4-1O11-2, O11T-1…

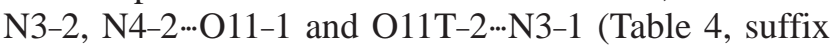
of atom labels represents the number of molecule). This type of interaction was observed in the crystal of C-Trp, which has a common backbone of C-Tyr. The phenolic hydroxyl group (O16) of Molecule $\mathbf{1}$ is interacted with $\mathrm{O} 2$ atoms, though that of Molecule $\mathbf{2}$ forms no hydrogen bonding. This may contribute to discriminate independent molecules having a similar conformation. In addition to the hydrogen bonds between the nitrogen and oxygen atoms, the $\mathrm{C}-\mathrm{H}$... O hydrogen bonds are observed, and Fig. 3 shows the interaction mode. The intermolecular distances of H5 ...O9 and H6... 99 are shorter than the sum of the van der Waals radii of hydrogen and oxygen (Table 4), and two independent molecules form a complementary pair.

Table 1 Crystal and experimental data

Formula: $2\left(\mathrm{C}_{16} \mathrm{H}_{18} \mathrm{~N}_{4} \mathrm{O}_{5}\right)$
Formula weight: 692.684
Crystal system: monoclinic
Space group: $P 2_{1} \quad Z=2$
Radiation=Cu $\mathrm{K}_{\alpha}(\lambda=1.5418 \AA)$
$a=9.563(1) \AA$
$b=10.454(1) \AA$
$c=16.864(6) \AA$
$V=1683.1(6) \AA^{3}$
Crystal size: $0.7 \times 0.12 \times 0.05 \mathrm{~mm}^{3}$
Plate, colorless
$D_{\mathrm{x}}=1.367 \mathrm{~g} / \mathrm{cm}^{3}$
$F(000)=728 \quad \mu\left(\mathrm{Cu} \mathrm{K}_{\alpha}\right)=0.870 \mathrm{~mm}^{-1}$
No. of measured reflection=3032 $\quad \theta_{\text {max }}=62.6^{\circ}$
No. of reflection used $=2847$
No. of parameters $=451$
$R=0.0384\left(\right.$ on $\left.F^{2}\right) \quad w R=0.0980\left(\right.$ on $\left.F^{2}\right)$
$\Delta \rho_{\text {max }}=0.314 \mathrm{e} \AA^{-3} \quad \Delta \rho_{\min }=-0.213$ e $\AA^{-3}$
Measurement: Rigaku AFC $5 \mathrm{R} / \mathrm{RU} 200$
Program system: MSC/AFC diffractometer control software
Structure determination: $\mathrm{SHELXS-97}$
Refinement: $\mathrm{SHELXL-97}$


Table 2 Final atomic coordinates and equivalent isotropic temperature factors for non-hydrogen atoms

\begin{tabular}{|c|c|c|c|c|c|c|c|c|c|}
\hline Atom & $x$ & $y$ & $z$ & $U_{\mathrm{eq}} / \AA^{2}$ & Atom & $x$ & $y$ & $z$ & $U_{\text {eq }} / \AA^{2}$ \\
\hline Molecule 1 & & & & & Molecule 2 & & & & \\
\hline N1 & $0.8465(2)$ & 0.2325 & $0.9799(1)$ & $0.0372(5)$ & N1 & $-0.1357(3)$ & $0.4142(3)$ & $1.2997(1)$ & $0.0427(6)$ \\
\hline $\mathrm{C} 2$ & $0.7386(3)$ & $0.2782(3)$ & $1.0235(2)$ & $0.0373(6)$ & $\mathrm{C} 2$ & $-0.1623(3)$ & $0.3733(3)$ & $1.3752(2)$ & $0.0433(7)$ \\
\hline $\mathrm{O} 2$ & $0.7378(2)$ & $0.3906(2)$ & $1.0445(1)$ & $0.0476(5)$ & $\mathrm{O} 2$ & $-0.2189(3)$ & $0.2710(3)$ & $1.3863(2)$ & $0.0658(7)$ \\
\hline N3 & $0.6366(3)$ & $0.1959(3)$ & $1.0424(1)$ & $0.0423(6)$ & N3 & $-0.1211(3)$ & $0.4521(3)$ & $1.4367(1)$ & $0.0424(6)$ \\
\hline $\mathrm{C} 4$ & $0.6351(3)$ & $0.0741(4)$ & $1.0171(2)$ & $0.0466(7)$ & $\mathrm{C} 4$ & $-0.0535(3)$ & $0.5635(3)$ & $1.4274(2)$ & $0.0455(7)$ \\
\hline N4 & $0.5323(4)$ & $0.0007(4)$ & $1.0383(2)$ & $0.073(1)$ & N4 & $-0.0154(4)$ & $0.6292(3)$ & $1.4903(2)$ & $0.0618(8)$ \\
\hline $\mathrm{C} 5$ & $0.7421(3)$ & $0.0287(4)$ & $0.9693(2)$ & $0.0516(8)$ & $\mathrm{C} 5$ & $-0.0316(4)$ & $0.6048(4)$ & $1.3485(2)$ & $0.0585(9)$ \\
\hline C6 & $0.8443(3)$ & $0.1099(3)$ & $0.9529(2)$ & $0.0452(8)$ & C6 & $-0.0742(4)$ & $0.5295(4)$ & $1.2885(2)$ & $0.0518(8)$ \\
\hline C7 & $0.9629(3)$ & $0.3199(3)$ & $0.9640(2)$ & $0.0406(7)$ & $\mathrm{C} 7$ & $-0.1717(3)$ & $0.3283(4)$ & $1.2325(2)$ & $0.0479(8)$ \\
\hline $\mathrm{C} 8$ & $0.9225(3)$ & $0.4173(3)$ & $0.9003(2)$ & $0.0416(7)$ & $\mathrm{C} 8$ & $-0.0633(3)$ & $0.2230(4)$ & $1.2260(2)$ & $0.0457(7)$ \\
\hline C9 & $0.8849(3)$ & $0.3534(3)$ & $0.8218(2)$ & $0.0372(6)$ & C9 & $0.0781(3)$ & $0.2801(3)$ & $1.2091(2)$ & $0.0399(7)$ \\
\hline O9 & $0.9404(3)$ & $0.2530(3)$ & $0.8036(1)$ & $0.0628(7)$ & O9 & $0.0896(2)$ & $0.3561(3)$ & $1.1546(1)$ & $0.0625(7)$ \\
\hline N10 & $0.7883(3)$ & $0.4108(3)$ & $0.7745(1)$ & $0.0390(5)$ & N10 & $0.1852(2)$ & $0.2477(3)$ & $1.2584(1)$ & $0.0402(6)$ \\
\hline $\mathrm{C} 10 \mathrm{~A}$ & $0.7448(3)$ & $0.3580(3)$ & $0.6979(2)$ & $0.0398(6)$ & $\mathrm{C} 10 \mathrm{~A}$ & $0.3238(3)$ & $0.2981(3)$ & $1.2458(2)$ & $0.0421(7)$ \\
\hline $\mathrm{C} 11$ & $0.8172(3)$ & $0.4206(3)$ & $0.6298(2)$ & $0.0403(7)$ & $\mathrm{C} 11$ & $0.3878(3)$ & $0.2337(3)$ & $1.1753(2)$ & $0.0429(7)$ \\
\hline O11 & $0.8546(3)$ & $0.5338(2)$ & $0.6332(1)$ & $0.0506(6)$ & O11 & $0.3609(3)$ & $0.1245(3)$ & $1.1557(2)$ & $0.0575(6)$ \\
\hline $\mathrm{O} 11 \mathrm{~T}$ & $0.8308(3)$ & $0.3481(2)$ & $0.5704(1)$ & $0.0602(7)$ & $\mathrm{O} 11 \mathrm{~T}$ & $0.4774(2)$ & $0.3049(2)$ & $1.1420(2)$ & $0.0619(7)$ \\
\hline $\mathrm{C} 12$ & $0.5850(3)$ & $0.3692(4)$ & $0.6825(2)$ & $0.0505(8)$ & $\mathrm{C} 12$ & $0.4232(3)$ & $0.2791(4)$ & $1.3196(2)$ & $0.0578(9)$ \\
\hline $\mathrm{C} 13$ & $0.4992(3)$ & $0.3055(3)$ & $0.7447(2)$ & $0.0429(7)$ & $\mathrm{C} 13$ & $0.3784(3)$ & $0.3449(4)$ & $1.3943(2)$ & $0.0515(8)$ \\
\hline $\mathrm{C} 14$ & $0.5365(4)$ & $0.1911(4)$ & $0.7808(2)$ & $0.0544(8)$ & $\mathrm{C} 14$ & $0.3380(4)$ & $0.2766(4)$ & $1.4581(2)$ & $0.063(1)$ \\
\hline C15 & $0.4518(4)$ & $0.1313(4)$ & $0.8330(2)$ & $0.0547(8)$ & $\mathrm{C} 15$ & $0.3006(5)$ & $0.3361(5)$ & $1.5269(2)$ & $0.068(1)$ \\
\hline $\mathrm{C} 16$ & $0.3281(4)$ & $0.1874(4)$ & $0.8515(2)$ & $0.0533(8)$ & $\mathrm{C} 16$ & $0.3016(4)$ & $0.4664(4)$ & $1.5323(2)$ & $0.060(1)$ \\
\hline O16 & $0.2384(3)$ & $0.1344(3)$ & $0.9031(2)$ & $0.083(1)$ & O16 & $0.2623(4)$ & $0.5198(3)$ & $1.6020(2)$ & $0.087(1)$ \\
\hline $\mathrm{C} 18$ & $0.3758(4)$ & $0.3592(4)$ & $0.7641(3)$ & $0.068(1)$ & $\mathrm{C} 18$ & $0.3407(5)$ & $0.5370(4)$ & $1.4697(3)$ & $0.076(1)$ \\
\hline $\mathrm{C} 17$ & $0.2906(4)$ & $0.3015(4)$ & $0.8167(3)$ & $0.077(1)$ & $\mathrm{C} 17$ & $0.3808(6)$ & $0.4766(5)$ & $1.4021(3)$ & $0.076(1)$ \\
\hline
\end{tabular}

$U_{\mathrm{eq}}=(1 / 3) \Sigma_{i} \Sigma_{j} U_{i j}\left(a_{i}^{*} a_{j}^{*}\right)\left(\boldsymbol{a}_{i} \cdot \boldsymbol{a}_{j}\right)$.

Table 3 Selected bond distances $(\AA)$ and torsion angles $\left({ }^{\circ}\right)$ for molecule $\mathbf{1}$ / molecule 2 .

\begin{tabular}{llll}
\hline N1-C7 & $1.476(3) / 1.472(4)$ & N10-C10A & $1.444(4) / 1.453(4)$ \\
C7-C8 & $1.514(4) / 1.520(5)$ & C10A-C11 & $1.522(4) / 1.525(4)$ \\
C8-C9 & $1.509(4) / 1.519(4)$ & C11-O11 & $1.237(4) / 1.212(4)$ \\
C9-O9 & $1.223(4) / 1.226(4)$ & C11-O11T & $1.269(4) / 1.288(4)$ \\
C9-N10 & $1.330(4) / 1.326(4)$ & & \\
C7-C8-C9-N10 & $148.2(3) / 125.7(3)$ & C10A-C12-C13-C14 & $-37.9(4) / 112.5(4)$ \\
C9-N10-C10A-C11 & $-98.4(3) /-73.7(3)$ & C10A-C12-C13-C18 & $145.1(3) /-69.6(5)$ \\
\hline
\end{tabular}

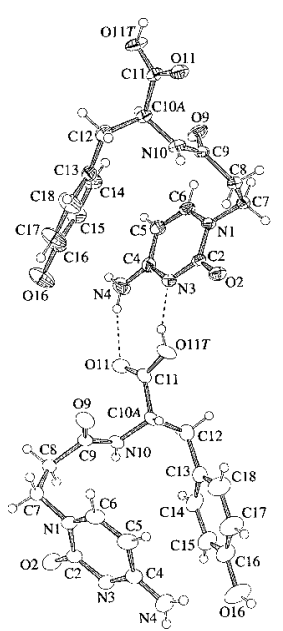

Fig. 2 ORTEP drawing of C-Tyr with atom labelings. The displacement ellipsoids are drawn at the $50 \%$ probability level. The italic numbers represent crystallographically independent Molecules $\mathbf{1}$ and 2, respectively.

This study was supported by a Grant-in-Aid for Scientific Research (07672427) from the Ministry of Education, Science, Sports and Culture.

\section{References}

1. K. Kamiichi, M. Doi, M. Nabae, T. Ishida and M. Inoue, $J$.
Table 4 Selected possible hydrogen bonds*

\begin{tabular}{|c|c|c|c|}
\hline $\mathrm{D}-\mathrm{H} \cdots \mathrm{A}$ & $\mathrm{D} \cdots \mathrm{A}(\AA)$ & $\mathrm{H} \cdots \mathrm{A}(\AA)$ & $\angle \mathrm{D}-\mathrm{H} \cdots \mathrm{A}\left({ }^{\circ}\right)$ \\
\hline 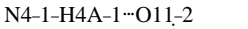 & $2.940(5)$ & 2.100 & 164.9 \\
\hline 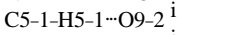 & $3.254(4)$ & 2.632 & 124.8 \\
\hline 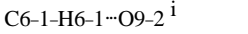 & $3.294(4)$ & 2.689 & 123.3 \\
\hline O11T-2-H11T-2-*N3-1 & $2.593(4)$ & 1.811 & 159.0 \\
\hline 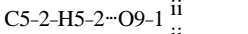 & $3.160(5)$ & 2.585 & 120.6 \\
\hline C6-2-H6-2 $\cdots$ O9-1 ${ }^{11} \quad \ldots$ & $3.119(5)$ & 2.471 & 126.9 \\
\hline O11T-1-H11T-1 N.N3-2 iii & $2.567(3)$ & 1.811 & 152.6 \\
\hline $\mathrm{O} 16-1-\mathrm{H} 16-1 \cdots \mathrm{O} 2-1{ }^{\mathrm{i}}$ & $2.703(4)$ & 1.795 & 163.6 \\
\hline
\end{tabular}

Symmetry code: (i) $1-x, y-1 / 2,2-z$, (ii) $1-x, y+1 / 2,2-z$, (iii) $\mathrm{x}+1, \mathrm{y}, \mathrm{z}-1$. *The suffixs ( 1 and 2 ) of atom labels correspond to the crystallographically independent molecules $\mathbf{1}$ and $\mathbf{2}$, respectively.

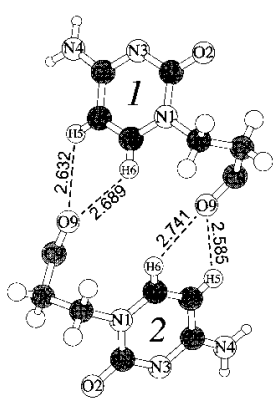

Fig. 3 Observed C-H...O interaction mode. The amino acid moiety is omitted for clarity. The italic numbers represent crystallographically independent Molecules $\mathbf{1}$ and 2, respectively. Molecule 2 is operated by the symmetry of (1-x, $y-1 / 2,2-z)$.

Chem. Soc., Perkin Trans. II, 1739 (1987).

2. M. Doi, M. Tarui, M. Ogata, A. Asano and T. Ishida, Acta Crystallogr. Sect. $C$, in press.

3. T. Ishida, H. Iyo, H. Ueda, M. Doi and M. Inoue, J. Chem. Soc., Chem. Commun., 217 (1990). 